DOI: $10.4274 /$ jarem.galenos.2020.3563

J Acad Res Med 2020;10(3):241-5

\title{
Optimal Target in Deep Brain Stimulation for Parkinson's Disease: Comparison of Atlas and Magnetic Resonance Imaging-based Stereotactic Targeting
}

\author{
(1) İdris Sertbaş \\ İstanbul Yeni Yüzyıl University Faculty of Medicine, Department of Neurosurgery, İstanbul, Turkey
}

Cite this article as: Sertbaş I. Optimal Target in Deep Brain Stimulation for Parkinson's Disease: Comparison of Atlas and Magnetic Resonance Imaging-based Stereotactic Targeting. J Acad Res Med 2020;10(3):241-5

\begin{abstract}
Objective: We aimed to compare magnetic resonance imaging (MRI) and atlas measurements to determine the target coordinates of globus pallidus internus (GPi) and subthalamic nucleus (STN) during stereotactic neurosurgery.

Methods: Eleven patients treated with bilateral deep brain stimulation (DBS) GPi and STN for the treatment of Parkinson's disease (PD) were included in the study. The target was chosen by direct visual recognition of GPi and STN in three-dimensional MRI. The coordinates were automatically saved using special software and converted to the anterior commissure-posterior commissure (AC-PC) coordinate system using a matrix conversion process. The same GPi and STN targets were identified based on the locations of brain structures shown in the Schaltenbrand atlases. MRI-based GPi and STN target coordinates were statistically compared with the corresponding atlas-based coordinates.

Results: Eleven patients were included in our study. The median age was $66.6 \pm 11.72$ in the GPi group and $47.50 \pm 14.20$ in the STN group. The average length of the AC-PC line was 26.15 \pm 1.42 in the STN group; and 26.46 \pm 1.34 in the GPi group. It was quite similar in both groups for each coordinate measurement, and the Intra-class Correlation Coefficients of each measurement were over $90 \%$.

Conclusion: According to the results of our study, target coordinates obtained by direct visual targeting on MRI and target coordinates obtained by indirect targeting based on atlas were highly compatible. The coordinates used for DBS in the treatment of PD were confirmed by both methods.

Keywords: MRI, deep brain stimulation, stereotactic targeting, subthalamic nucleus, globus pallidus
\end{abstract}

\section{INTRODUCTION}

Parkinsonism is a clinical syndrome that occurs with any combination of bradykinesia, resting tremor, rigidity, and postural instability. The most common form of parkinsonism is Parkinson's disease (PD); a chronic, progressive disease caused by the degenerative loss of dopaminergic neurons in the brain and characterized by clinically asymmetric parkinsonism (1). During diagnosis and treatment, many factors should be carefully evaluated, including the patient's signs, symptoms, age, stage of the disease, degree of functional disease, level of physical activity and efficiency (2). Treatment of PD can be divided into pharmacological, non-pharmacological and surgical treatment.

Deep brain stimulation (DBS) is the most commonly performed surgical procedure for the treatment of advanced PD (3). Evidence from randomized controlled trials suggests that either the DBS of the subthalamic nucleus (STN) or the internal globus pallidus (GPi) mitigate motor fluctuations and dyskinesia associated with

ORCID ID of the author: i.S. 0000-0002-6904-6678. 
advanced PD. The STN and GPi are two of the most common target structures used for DBS $(3,4)$. The lead should be placed correctly to maximize therapeutic benefit and minimize side effects. Preoperative localization of target structures can be performed directly from stereotactic preoperative magnetic resonance imaging (MRI) or indirectly from atlas coordinates and predefined anatomical markers. Given the anatomical variability of the target core between patients and a degree of atrophy that can be found in patients with neurodegenerative diseases, the direct targeting method is unquestionably more suitable for individual patients. However, this technique can result in limited contrast and relatively low visibility of target points in standard MRI $(5,6)$.

Technological advances in imaging methods have facilitated direct target planning and post-operative lead localization. But intraoperative verification of the electrode position relative to the intended target coordinates is difficult. Although there are very few published data documenting the best targeting method for DBS and the accuracy of any electrode placement method, there is no consensus on the subject (7-10). The aim of this study was to investigate the reliability of atlas-derived data by comparing it with direct targeting on MRI, and to assess the suitability of each technique for stereotactic targeting.

\section{METHODS}

This study was conducted in accordance with the principles of the Helsinki Declaration of the World Medical Association "Ethical Principles for Medical Research Involving Human Subjects" (held in October 2013). Ethics committee approval for this study was obtained from İstanbul Yeni Yüzyll University Clinical Research Ethics Committee (approval number: 2020/02, date: 10.02.2020).

This study included 11 patients admitted to a neurosurgery clinic for drug-resistant PD and underwent DBS. Patients underwent bilateral electrode implantation for continuous stimulation of GPi $(n=5)$ and STN $(n=6)$ under local anesthesia.

Under local anesthesia, the stereotactic frame (Zamorano-Dujovny open ceramic version, Stryker Leibinger, Freiburg, Germany) was placed parallel to the anterior commissure-posterior commissure (AC-PC) line relative to the external landmarks (lower orbital ring, external auditory canal). AC-PC coordinates were determined on axial T1-weighted images (T1-WI) using MRI, Achieva 1.5 Tesla (Philips, Best, Netherlands), and the coordinates of the midcommistatic point (AC-PC) and AC-PC distance were calculated accordingly. The location of the STN and GPi cores was found and their coordinates were calculated according to the AC-PC line. Schaltenbrand-Wahren-Atlas was also used as AC and PC reference points and merged with a volumetric T1-WI MRI data set.

The target was then chosen based on the Schaltenbrand atlas (11). In this atlas, one of the GPi and STN cores was selected, the distances between the $A C$ and $P C$ midpoints were measured on the Schaltenbrand atlas after the target was detected. According to the AC-PC line on the atlas; vertical, inferior and anterioposterior coordinates were determined and the plate where the DBS target was wanted to be placed was selected.

Then, in the operating room, a stereotactic electrode guidance device was mounted and a $14 \mathrm{~mm}$ hole was drilled at the predetermined orbital level. Microelectrode recording was not used. Electrode implantation was performed. Distal electrode tips were placed subcutaneously. Under general anesthesia, the brain pacemaker placed under the skin with iv propofol and remifentanil was connected to the electrodes. Computed Tomography was performed to control post-operative patients in terms of possible complications.

\section{Statistical Analysis}

All analyzes were done in SPSS v21 (SPSS Inc., Chicago, IL, USA). Due to the small sample size, the coordinates were analyzed with the Wilcoxon signed-ranks test. The fit of the coordinates for both methods was evaluated using Cronbach's Alpha and Intra-class Correlation Coefficient (ICC). Accordingly, we suggest that ICC values below 0 indicate "low" reliability; values between 0.5 and 0.75 indicate "moderate" reliability; values between 0.75 and 0.9 indicate "good" reliability; and values greater than 0.90 indicate "excellent" reliability (12). P $<0.05$ was considered statistically significant.

\section{RESULTS}

Eleven patients ( 4 females, 7 males) were included in our study. The mean age was $66.6 \pm 11.72$ in the GPi group and $47.50 \pm 14.20$ in the STN group.

The average length of the AC-PC line was $26.15 \pm 1.42$ in the STN group. The target coordinates set in Atlas and MRI for STN showed "excellent" correlation for distances between right hemisphere $d x, d z$ and left hemisphere $d x, d y, d z$ ( $p$-value for all $>0.05$ and ICC $>0.90$ ) (Table 1).

The average length of the AC-PC line was $26.46 \pm 1.34$ in the GPi group. The target coordinates set in Atlas and MRI for GPi showed "excellent" correlation in distances between right hemisphere $\mathrm{dx}$, $d y, d z$ and left hemisphere $d x, d z$ ( $p$-value for all $>0.05$ and ICC $>0.90$ ) (Table 2).

\section{DISCUSSION}

According to the results of our study, the target coordinates we determined in Atlas and MRI for STN and GPi showed "excellent" correlation. Accordingly, DBS structures, which are often not clearly visualized using Atlas, have been verified by MRI techniques.

Reliable identification of the anatomical boundaries of STN and GPi is a critical step for DBS of these structures. Atlas-based coordinates are limited to relying only on a few brain samples, and several studies have documented inter-individual variations in the position of these nuclei. The Schaltenbrand atlas consists of successive brain slices (plates) obtained from a brain. The coordinates calculated based on the structures found in this atlas 
Table 1. Distribution of STN coordinates according to patient characteristics

\begin{tabular}{|c|c|c|c|c|c|c|c|c|}
\hline & Mean & SD & Median & Minimum & Maximum & $p$ & Cronbach's Alpha & ICC \\
\hline Age & 47.50 & 14.20 & 47.50 & 31.00 & 69.00 & - & - & - \\
\hline AC-PC distance & 26.15 & 1.42 & 26.30 & 23.80 & 27.60 & - & - & - \\
\hline \multicolumn{9}{|l|}{ Right hemisphere } \\
\hline \multicolumn{9}{|l|}{$d x$} \\
\hline Atlas & 11.82 & 2.13 & 11.15 & 9.70 & 15.90 & \multirow{2}{*}{0.109} & \multirow{2}{*}{0.995} & \multirow{2}{*}{0.992} \\
\hline MRI & 12.08 & 2.29 & 11.50 & 10.00 & 16.50 & & & \\
\hline \multicolumn{9}{|l|}{ dy } \\
\hline Atlas & 2.60 & 2.78 & 1.70 & 0.40 & 8.00 & \multirow{2}{*}{0.043} & \multirow{2}{*}{0.985} & \multirow{2}{*}{0.968} \\
\hline MRI & 3.33 & 2.34 & 2.50 & 2.00 & 8.00 & & & \\
\hline \multicolumn{9}{|l|}{$d z$} \\
\hline Atlas & 3.67 & 1.36 & 3.80 & 1.40 & 5.00 & \multirow{2}{*}{1.000} & \multirow{2}{*}{0.992} & \multirow{2}{*}{0.993} \\
\hline MRI & 3.67 & 1.51 & 4.00 & 1.00 & 5.00 & & & \\
\hline \multicolumn{9}{|l|}{ Left hemisphere } \\
\hline \multicolumn{9}{|l|}{$d x$} \\
\hline Atlas & 11.58 & 2.76 & 11.00 & 9.00 & 16.60 & \multirow{2}{*}{0.078} & \multirow{2}{*}{0.992} & \multirow{2}{*}{0.984} \\
\hline MRI & 12.08 & 2.46 & 11.50 & 10.00 & 16.50 & & & \\
\hline \multicolumn{9}{|l|}{ dy } \\
\hline Atlas & 2.28 & 2.00 & 1.80 & 0.30 & 5.90 & \multirow{2}{*}{0.176} & \multirow{2}{*}{0.981} & \multirow{2}{*}{0.977} \\
\hline MRI & 2.58 & 1.69 & 2.50 & 1.00 & 5.50 & & & \\
\hline \multicolumn{9}{|l|}{$d z$} \\
\hline Atlas & 3.03 & 2.06 & 3.55 & 0.30 & 5.00 & \multirow{2}{*}{0.109} & \multirow{2}{*}{0.989} & \multirow{2}{*}{0.985} \\
\hline MRI & 3.33 & 1.86 & 4.00 & 1.00 & 5.00 & & & \\
\hline
\end{tabular}

\section{Table 2. Distribution of GPi coordinates according to patient characteristics}

\begin{tabular}{|c|c|c|c|c|c|c|c|c|}
\hline & Mean & SD & Median & Minimum & Max & $p$ & Cronbach's Alpha & ICC \\
\hline Age & 66.60 & 11.72 & 71.00 & 48.00 & 78.00 & - & - & - \\
\hline AC-PC distance & 26.46 & 1.34 & 26.50 & 25.10 & 28.20 & - & - & - \\
\hline \multicolumn{9}{|l|}{ Right hemisphere } \\
\hline \multicolumn{9}{|l|}{$d x$} \\
\hline Atlas & 17.34 & 2.95 & 15.70 & 15.00 & 22.10 & \multirow{2}{*}{0.080} & \multirow{2}{*}{0.988} & \multirow{2}{*}{0.978} \\
\hline MRI & 18.00 & 2.92 & 17.00 & 16.00 & 23.00 & & & \\
\hline \multicolumn{9}{|l|}{ dy } \\
\hline Atlas & 6.56 & 2.76 & 7.90 & 2.20 & 8.90 & \multirow{2}{*}{0.223} & \multirow{2}{*}{0.991} & \multirow{2}{*}{0.990} \\
\hline MRI & 6.80 & 2.68 & 8.00 & 3.00 & 9.00 & & & \\
\hline \multicolumn{9}{|l|}{$d z$} \\
\hline Atlas & 2.12 & 1.18 & 1.70 & 1.00 & 3.70 & \multirow{2}{*}{0.593} & \multirow{2}{*}{0.949} & \multirow{2}{*}{0.958} \\
\hline MRI & 2.20 & 1.30 & 2.00 & 1.00 & 4.00 & & & \\
\hline \multicolumn{9}{|l|}{ Left hemisphere } \\
\hline \multicolumn{9}{|l|}{$d x$} \\
\hline Atlas & 17.36 & 2.93 & 15.70 & 15.10 & 22.10 & \multirow{2}{*}{0.078} & \multirow{2}{*}{0.989} & \multirow{2}{*}{0.979} \\
\hline MRI & 18.00 & 2.92 & 17.00 & 16.00 & 23.00 & & & \\
\hline \multicolumn{9}{|l|}{ dy } \\
\hline Atlas & 6.48 & 2.65 & 7.60 & 2.30 & 8.90 & \multirow{2}{*}{0.043} & \multirow{2}{*}{0.997} & \multirow{2}{*}{0.987} \\
\hline MRI & 7.00 & 2.55 & 8.00 & 3.00 & 9.00 & & & \\
\hline \multicolumn{9}{|l|}{$d z$} \\
\hline Atlas & 2.12 & 1.16 & 1.70 & 1.00 & 3.60 & \multirow{2}{*}{0.144} & \multirow{2}{*}{0.976} & \multirow{2}{*}{0.966} \\
\hline MRI & 2.40 & 1.14 & 2.00 & 1.00 & 4.00 & & & \\
\hline
\end{tabular}


are not obtained from an average brain, but from a photograph that actually corresponds to a slice of a certain thickness (13). It has been recognized that the use of atlases for stereotactic neurosurgery is conditioned on data normalization according to the characteristics of each brain. However, there has been no consensus on the normalization process $(14,15)$.

MRI allows excellent visualization of commissures, which are thalamic organization. It also shows individual anatomical variations while reducing the imaging artifacts produced by the stereotactic framework. It is possible to obtain millimeter sections with good signal with 3-D gradient-Echo reception. It allows the radiologist to position all anatomical landmarks identified by high-resolution MRI ventriculography in a non-invasive manner. In addition, structures not seen in ventriculograms, such as the internal capsule, can be visualized $(16,17)$. There are concerns that artifacts on MRI will distract intracranial targets from their actual anatomical position, leading to errors in determining target coordinates and, as a result, failed stereotactic procedures. However, precise MRI-guided stereotactic procedures can be performed using high-field MRI with a homogeneous magnetic field and linear field gradients $(18,19)$. In a study conducted using anatomical samples, mean stereotactic errors were reported to be $0.48 \pm 0.17 \mathrm{~mm} ; 0.69 \pm 0.14 \mathrm{~mm}$ and $0.82 \pm 0.13 \mathrm{~mm}$, respectively, in the $x, y$ and $z$ directions (18). Other studies that report the reliability of direct MRI coordinates are mainly based on visualizing the structure only in the coronal plane and do not target a specific subregion $(20,21)$. In another study comparing MRI and Atlas methods for DBS in Parkinson's patients, it was concluded that high-resolution MRI, which enables direct visualization of the nucleus in both axial and coronal planes, correlates borders in both planes and creates a three-dimensional structure would be more effective (7). These results support that a functional MRI procedure can be performed safely after its accuracy has been verified by a certain stereotactic MRI standardization. A recent metaanalysis concluded that STN for DBS is difficult due to low resolution and geometric distortion in MRI sequences used in direct targeting, but that STN boundaries can be better defined with new MRI techniques. Accordingly, sensitivity-based imaging techniques and image reconstruction methods can show the way to produce high-quality, artifact-free images that neurosurgeons can use to accurately and reliably target their electrodes (22).

\section{Study Limitations}

Our study also had limitations. It is a retrospective study in nature, based on patient file records. Although the patient population is not sufficient, objective results can be obtained with prospective studies.

\section{CONCLUSION}

In light of these results, normalizing Atlas data based on the reference of more patients will bring Atlas coordinates closer to MRI targets. In addition, increased image quality along with the developing technology in the field of MRI will also allow sterotactic targeting to be performed more accurately and in a standard way. Based on the overlap of the target coordinates we determined in our study in both Atlas and MRI, we believe that the targets we determined will be a guide for subsequent studies. Randomizedcontrolled studies are needed on this issue.

The target coordinates we set for DBS coincide with each other in both Atlas and MRI. Due to atrophy in neurodegenerative conditions, the anatomy of patients will also be different. Therefore, a direct targeting technique based on the patient's own brain anatomy may be a more convenient way of preoperative targeting for DBS. Accurate anatomical targeting can also minimize the number of exploration marks required for physiological testing of the target, thus enabling faster and safer surgery.

Ethics Committee Approval: Ethics committee approval for this study was obtained from İstanbul Yeni Yüzyıl University Clinical Research Ethics Committee (approval number: 2020/02, date: 10.02.2020).

Informed Consent: Due to the retrospective design of the study, patient consent was not obtained.

Peer-review: Externally peer-reviewed.

Financial Disclosure: The author declared that this study has received no financial support.

\section{REFERENCES}

1. Watts RL, Koller WC. Movement disorders: neurologic principles \& practice. McGraw-Hill Professional, 2004.

2. Odin P, Chaudhuri KR, Slevin J, Volkmann J, Dietrichs E, MartinezMartin $\mathrm{P}$, et al. Collective physician perspectives on non-oral medication approaches for the management of clinically relevant unresolved issues in Parkinson's disease: consensus from an international survey and discussion program. Parkinsonism Relat Disord 2015; 21: 1133-44.

3. Fasano A, Daniele A, Albanese A. Treatment of motor and non-motor features of Parkinson's disease with deep brain stimulation. Lancet Neurol 2012; 11: 429-42.

4. Deuschl G, Schade-Brittinger C, Krack P, Volkmann J, Schäfer H, Bötzel $K$, et al. A randomized trial of deep-brain stimulation for Parkinson's disease. N Eng J Med 2006; 355: 896-908.

5. Odekerken VJ, Van Laar T, Staal MJ, Mosch A, Hoffmann CF, Nijssen PC, et al. Subthalamic nucleus versus globus pallidus bilateral deep brain stimulation for advanced Parkinson's disease (NSTAPS study): a randomised controlled trial. Lancet Neurol 2013; 12: 37-44.

6. Öztürk S, Kocabiçak E. Parkinson hastalığında nöromodülasyon. Turkiye Klinikleri Phys Med Rehab 2018; 11: 23-32.

7. Vayssiere N, Hemm S, Cif L, Picot MC, Diakonova N, El Fertit H, et al. Comparison of atlas-and magnetic resonance imaging-based stereotactic targeting of the globus pallidus internus in the performance of deep brain stimulation for the treatment of dystonia. J Neurosurg 2002; 96: 673-9.

8. Pinsker M, Volkmann J, Falk D, Herzog J, Steigerwald F, Deuschl G, et al. Deep brain stimulation of the internal globus pallidus in dystonia: target localisation under general anaesthesia. Acta neurochirurgica (Wien) 2009; 151: 751-8.

9. Schlaier J, Schoedel P, Lange M, Winkler J, Warnat J, Dorenbeck U, et al. Reliability of atlas-derived coordinates in deep brain stimulation. Acta Neurochirurgica 2005; 147: 1175-80

10. Tolleson C, Pallavaram S, Li C, Fang J, Phibbs F, Konrad F, et al. The optimal pallidal target in deep brain stimulation for dystonia: a study using a functional atlas based on nonlinear image registration. Stereotact Funct Neurosurg 2015; 93: 17-24.

11. Schaltenbrand G. Atlas for stereotaxy of the human brain. Georg Thieme 1977.

12. Koo TK, Li MY. A guideline of selecting and reporting intraclass correlation coefficients for reliability research. J Chiropr Med 2016; 15: 155-63. 
13. Bajcsy R, Lieberson R, Reivich M. A computerized system for the elastic matching of deformed radiographic images to idealized atlas images. $J$ Comput Assist Tomogr 1983; 7: 618-25.

14. Lancaster JL, Fox PT, Downs H, Nickerson DS, Hander TA, El Mallah M, et al. Global spatial normalization of human brain using convex hulls. J Nucl Med 1999; 40: 942-55.

15. Davatzikos C. Spatial normalization of $3 \mathrm{D}$ brain images using deformable models. J Comput Assist Tomogr 1996; 20: 656-65.

16. Aviles-Olmos I, Kefalopoulou Z, Tripoliti E, Candelario J, Akram H, Martinez-Torres I, et al. Long-term outcome of subthalamic nucleus deep brain stimulation for Parkinson's disease using an MRI-guided and MRIverified approach. J Neurol Neurosurg Psychiatr 2014; 85: 1419-25.

17. Brunenberg EJ, Platel B, Hofman PA, ter Haar Romeny BM, VisserVandewalle $\mathrm{V}$. Magnetic resonance imaging techniques for visualization of the subthalamic nucleus: a review. J Neurosurg 2011; 115: 971-84.

18. Dormont $D$, Zerah $M$, Cornu $P$, Parker $F$, Aubert B, Sigal $R$, et al. A technique of measuring the precision of an MR-guided stereotaxic installation using anatomic specimens. American J Neuroradiol 1994; 15 : 365-71.

19. Kondziolka D, Dempsey PK, Lunsford LD, Kestle JR, Dolan EJ, Kanal E, et al. A comparison between magnetic resonance imaging and computed tomography for stereotactic coordinate determination. Neurosurgery 1992; 30: 402-7.

20. Zonenshayn M, Rezai AR, Mogilner AY, Beric A, Sterio D, Kelly PJ. Comparison of anatomic and neurophysiological methods for subthalamic nucleus targeting. Neurosurgery 2000; 47: 282-94.

21. Cuny E, Guehl D, Burbaud P, Gross C, Dousset V, Rougier A. Lack of agreement between direct magnetic resonance imaging and statistical determination of a subthalamic target: the role of electrophysiological guidance. J Neurosurgery 2002; 97: 591-7.

22. Chandran AS, Bynevelt $M$, Lind CR. Magnetic resonance imaging of the subthalamic nucleus for deep brain stimulation. J Neurosurgery 2016; 124: 96-105. 